\title{
Anisotropic Foams Via Frontal Polymerization
}

Diego M. Alzate-Sanchez ${ }^{1,2}$, Morgan M. Cencer ${ }^{1,2}$, Michael Rogalski ${ }^{3}$, Mariana Kersh ${ }^{1,3}$, Nancy Sottos, ${ }^{1,3}$ and Jeffrey S. Moore ${ }^{1,2 *}$

Affiliations: ${ }^{1 B e c k m a n}$ Institute for Advanced Science and Technology, University of Illinois at Urbana-Champaign, Urbana, Illinois 61801, United States. ${ }^{2}$ Department of Chemistry, University of Illinois at Urbana-Champaign, Urbana, Illinois 61801, United States. ${ }^{3}$ The Grainger College of Engineering, University of Illinois at Urbana-Champaign, Urbana, Illinois 61801, United States

${ }^{\star}$ Corresponding author. Email: jsmoore@illinois.edu

\section{Abstract}

The properties of foams, an important class of cellular solids, are most sensitive to the volume fraction and openness of its elementary compartments; size, shape, orientation, and the interconnectedness of

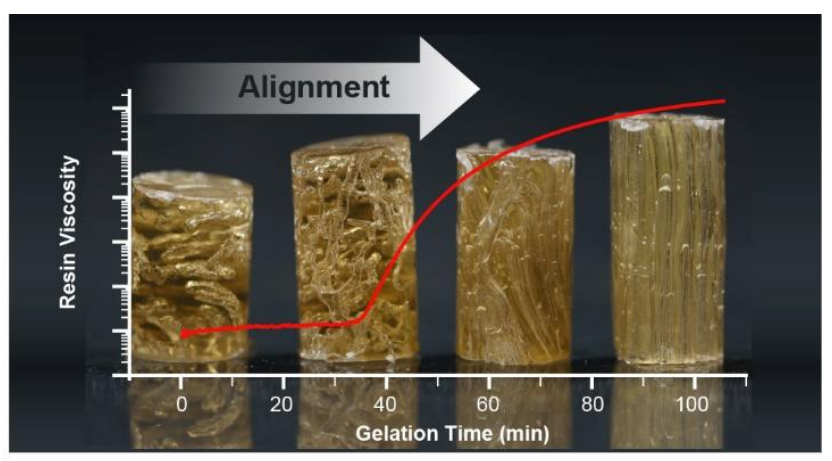
the cells are other important design attributes. Control of these morphological traits would allow the tailored fabrication of useful materials including highly porous solids, anisotropic heat conductors, tough composites, among others. While approaches like ice templating has produced foams with elongated cells, there is a need for rapid, versatile, and energy efficient methods that also control the local order and macroscopic alignment of cellular elements. Here we describe a fast and convenient method to obtain anisotropic structural foams using frontal polymerization. We fabricated foams by curing mixtures of dicyclopentadiene and a physical blowing agent via frontal ring opening metathesis polymerization (FROMP). The materials were characterized using micro-computed tomography and an image analysis protocol to quantify morphological characteristics including volume fraction and anisotropy. The cellular structure, porosity, and hardness 
of the foams changed with blowing agent, concentration, and resin viscosity. Moreover, we used a full factorial combination of variables to correlate each parameter with the structure of the obtained foams. We found a strong correlation between the resin viscosity and the foam's cellular structure. Furthermore, a specific combination of input parameters controlled the transitions from (i) isotropic to anisotropic cellular structures, (ii) porous to non-porous, and (iii) soft to hard foams. Our results demonstrate the controlled production of foams with specific morphologies using the simple and efficient method of frontal polymerization. This work shows promise for creating foams with aligned cellular structures that allow anisotropic mass and energy transport properties in high performance structural solids.

\section{Introduction}

Cellular solids are an assembly of small compartments whose edges or faces are packed together. Polymeric foams, a class of cellular solids, are employed in diverse applications such as protective packaging and thermal insulation. ${ }^{[1]}$ More recently, improvements in foaming processes, along with the incorporation of functional groups and novel architectures, have enabled the use of polymeric foams in more specialized applications, including tissue engineering and membrane separations. ${ }^{[2,3]}$ However, many polymeric foams are prepared with an isotropic cellular structure, ignoring unique characteristics that emerge from an anisotropic distribution of their compartments.

In many natural materials, hollow spaces form channeled structures, creating an anisotropic topology important for the transport of fluids that distribute nutrients and remove waste. ${ }^{[4]}$ Inspired by these biomaterials, the development of foams with a 
controlled cell shape and orientation will add useful properties to high-performance polymers. ${ }^{[5,6]}$ Control of the foam's cellular structure and topology has been achieved by methods like chemical vapor deposition, 3D printing, and freeze-casting. ${ }^{[7-9]}$ Anisotropic foams fabricated via freeze casting, where the foam's compartments are assembled into 3D architectures using growing crystals as templates, allows access to a range of porous architectures. ${ }^{[10]}$ Nonetheless, freeze casting requires specialized equipment, high energy inputs, postprocessing of the sample, and water-dispersible components. ${ }^{[11]}$ Therefore, development of a simple method to fabricate complex foam topologies is envisioned.

Frontal polymerization (FP), a bulk polymerization method where a self-sustained reaction is initiated by a locally applied triggering event, induces the curing of a polymer using the heat released from the reaction. ${ }^{[12]}$ The method rapidly and controllably transforms monomer to polymer, and is useful for creating composites, gradient materials, coatings, and resins for damage repair. ${ }^{[13,14]} \mathrm{FP}$ in combination with blowing agents produces cellular solids with tunable pore size and porosity in a synchronized polymerization-foaming process. ${ }^{[15,16]}$ However, the directional characteristics of FP have not yet been explored to control the morphology of porous materials.

Dicyclopentadiene (DCPD), a strained hydrocarbon monomer coproduced in large quantities during oil steam cracking, undergoes bulk phase olefin metathesis resulting in durable thermoset materials. ${ }^{[17]}$ FP of DCPD was introduced by Mariani et al. in 2001. ${ }^{[18]}$ Our group demonstrated the frontal ring opening metathesis polymerization (FROMP) 
curing of DCPD using a ruthenium catalyst and alkyl phosphite inhibitors. ${ }^{[19]}$ At room temperature, this system slowly transforms the monomer into a viscoelastic gel, which we previously used to manufacture free-standing objects via 3D printing. ${ }^{[20]}$ When the gel phase receives a brief and localized thermal stimulus, the FROMP reaction begins resulting in rapid curing. Once started, the heat of the reaction provides the thermal energy to thermally activate the catalyst sustaining the FROMP process without the need for external energy as typically required to cure conventional epoxy-based thermosets.

Two methods predominate for DCPD foam manufacturing; chemically induced phase separation, which produces open and close pore cells depending on the amount of solvent used during the polymerization, and high internal phase emulsion polymerization, for the fabrication of open pore cell structures and highly porous foams. ${ }^{[21]}$ However, frontal polymerization to manufacture anisotropic foams is largely unexplored.

We hypothesized a physicochemical rationale for the generation of anisotropic foams based on the thermomechanical gradient fields present at the FP liquid-to-solid boundary (Scheme 1, inset). The reaction front of DCPD typically experiences maximum temperatures in the range of $150-220^{\circ} \mathrm{C}$, temperature gradients as large as $0.1^{\circ} \mathrm{C} / \mu \mathrm{m},{ }^{[22]}$ and orders of magnitude change in viscosity over just a few microns. Void formation is expected from vaporized blowing driven by the localized temperature increase of the exothermic reaction. The moving front boundary feeds the nucleated voids with a continuous supply of vapor. The growing voids are expected to travel along the path of least resistance which is dictated by local variations in viscosity. This viscosity gradient is largest in the same direction that the reaction front propagates. Anisotropic channels 
aligned along the direction of the propagating front are thus postulated. In testing the thermomechanical foaming hypothesis, we discovered that the transition from an isotropic to an anisotropic cellular structure depends on the rheological properties of the polymerization mixture. The technique developed herein offers a simple, rapid, and energy efficient method to construct diverse foam morphologies for the design of multifunctional materials tunable for target-specific applications.

\section{Results and discussion}

Synthesis of porous poly-DCPD via FROMP

We prepared poly-DCPD foams based on a formulation containing DCPD, blowing agent, second-generation Grubbs catalyst (GC2), tributyl phosphite (TBP), and phenylcyclohexane $(\mathrm{PCH}){ }^{[19]}$ We mixed one equiv of DCPD with the blowing agent, followed by the addition of GC2 $\left(1 \times 10^{-4}\right.$ equiv per monomer $)$ and TBP $\left(1 \times 10^{-4}\right.$ equiv per monomer) dissolved in phenylcyclohexane (12 mM). This solution, without degassing it, was poured into glass test tubes and the bottom of the tube was briefly heated with a soldering iron to initiate frontal polymerization. The heat released from the ring opening of DCPD during polymerization simultaneously also induces a liquid-to-vapor phase change of the blowing agent. (Scheme 1). 


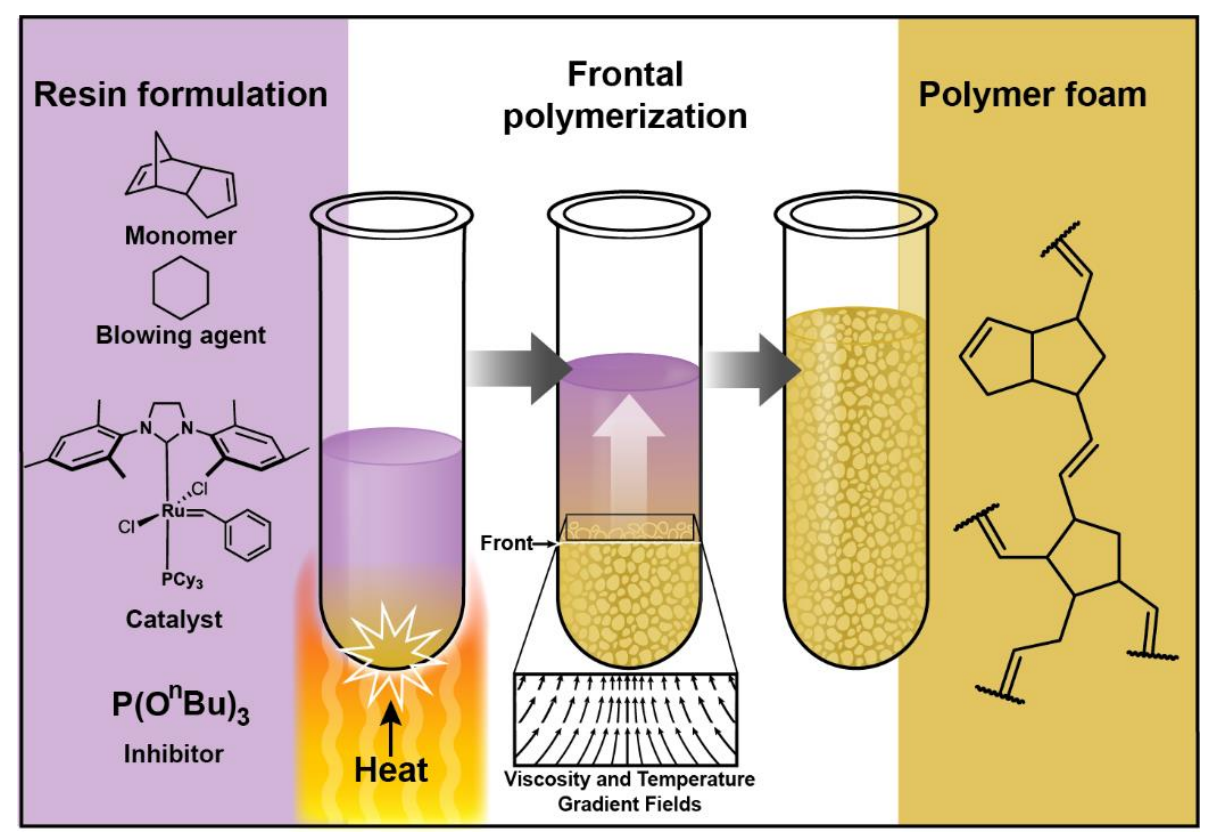

Scheme 1. Frontal Ring Opening Metathesis Polymerization of Dicyclopentadiene in the presence of blowing agents to obtain foams. Viscosity and temperature gradient fields (inset) are largest at the front boundary and aligned to the direction of the propagating front.

We surveyed six different blowing agents, with boiling points ranging from $36^{\circ} \mathrm{C}$ to 117 ${ }^{\circ} \mathrm{C}$, separately mixed with DCPD in a 1 to 4 weight ratio. All samples successfully underwent frontal polymerization (Figure 1a). Qualitatively we found that blowing agents with low boiling points like $n$-pentane and dichloromethane produced foams with irregular structures (Figure S1 and S2). In sharp contrast, blowing agents with elevated boiling points like n-butanol did not produce a continuous foam. Additionally, the polymer's opacity increases when using alcohols, presumably due to the poor miscibility of the polymer and the blowing agent (Figure S3). Our initial experiments demonstrated that incorporation of blowing agents during FROMP of DCPD generates foams, whose appearance and cellular structure depended on the blowing agent employed. 


\section{Foams Using Cyclohexane}

To study the foaming process in greater detail, we focused on the use of cyclohexane as the blowing agent. Initially, we evaluated the foam structure with cyclohexane amounts ranging from $10 \mathrm{wt} \%$ to $70 \mathrm{wt} \%$. (Figure $1 \mathrm{~b}$ ). We observed that initiation time, which is the heating time required for the reaction to become self-sustaining, increases as the blowing agent concentration increases to $20-30 \mathrm{wt} \%$ and approaches a limit at $50 \mathrm{wt} \%$ (Figure S4). Long initiation times decreased the concentration of the blowing agent in the resin before the polymerization was triggered. Concentrations above $30 \mathrm{wt} \%$ also produced uncontrollable evaporation, causing irregular foaming. We observed irregularities in the cellular structure of the foam when concentrations of cyclohexane were higher than 30 wt $\%$. Furthermore, more than $50 \mathrm{wt} \%$ of cyclohexane produced very soft foams with gellike structures due to incomplete evaporation of the blowing agent (Figure S5).

We studied the influence of the delay time between formulation mixing and reaction triggering using DCPD resins containing $20 \mathrm{wt} \%$ cyclohexane. During the delay time, the resin sits quiescently at room temperature all the while a slow background metathesis reaction ensues, causing a rise in viscosity, eventually leading to a soft gel. We refer to the delay time as the gelation time since it is during this period when gelation ensues. Initially, we observed spontaneous polymerization at 80 min of gelation time resulting in a non-porous solid. We found that the rate of viscosity change during the gelation period depends on the amount of butylated hydroxytoluene, an inhibitor present in the DCPD monomer, which varies depending on the commercial source. A systematic study of the gelation process is outside of the scope of the research described here (Figure S6). 
Practically, we found that the onset of spontaneous polymerization was delayed to 210 min by decreasing the amount of catalyst. The frontal velocity decreased when the gelation time increased because there is a lower energy release rate due to partial monomer consumption (Figure 1d). Additionally, resins polymerized at times higher than 180 min were nonporous, indicating that the thermal power produced by the chemical reaction did not efficiently vaporize the cyclohexane (Figure S7).

Foam morphology changed as a function of gelation time such that longer times yielded more controlled evolution of the blowing agent, which increased the alignment of the compartments created during foaming (Figure S8). To determine the influence of the gelation time on the cellular structure of the fabricated foams, we used X-ray microcomputed tomography to acquire images of the solids, followed by reconstruction and processing of the images using the imagej2 software (Figure 1c). ${ }^{[23]}$ We determined the anisotropy of the foam's cellular structure as a function of gelation time, using the bone $\mathrm{J}$ image analysis package, where an anisotropy value of zero indicates a completely isotropic sample and a value of unity indicates a prevailing orientation of the sample's cellular structure. ${ }^{[24]}$ We found a step-type correlation between the anisotropy and the gelation time, with a clear shift from moderate to high anisotropy at $60 \mathrm{~min}$, consistent with a higher alignment of the voids in the cellular structure. (Figure 1e). The prevailing direction of orientation is co-aligned to the direction of the propagating front. 
We observed an increase in resin viscosity as a function of gelation time. Therefore, we hypothesized that the pore alignment correlates with the resin's rheological properties. We employed oscillation rheology to determine the viscoelastic properties of the resin. Our results showed a sharp increase in the viscosity at 30 min of gelation time, which matches the observed increase in the cellular structure anisotropy of the foams (Figure 1f). Furthermore, the gel point obtained from the crossover of the storage and loss modulus was located at 25.9 min, indicating the resin had a gel-like character after this point (Figure S9). Our observations show that a higher viscosity and gel-like character of the resin was directly correlated to the anisotropy of the foams, indicating that the morphology of the foam cellular structure is influenced by the rheological properties of the resin. Based on the findings in this section, we observed that foam morphology depends on the blowing agent, concentration, and gelation time. 


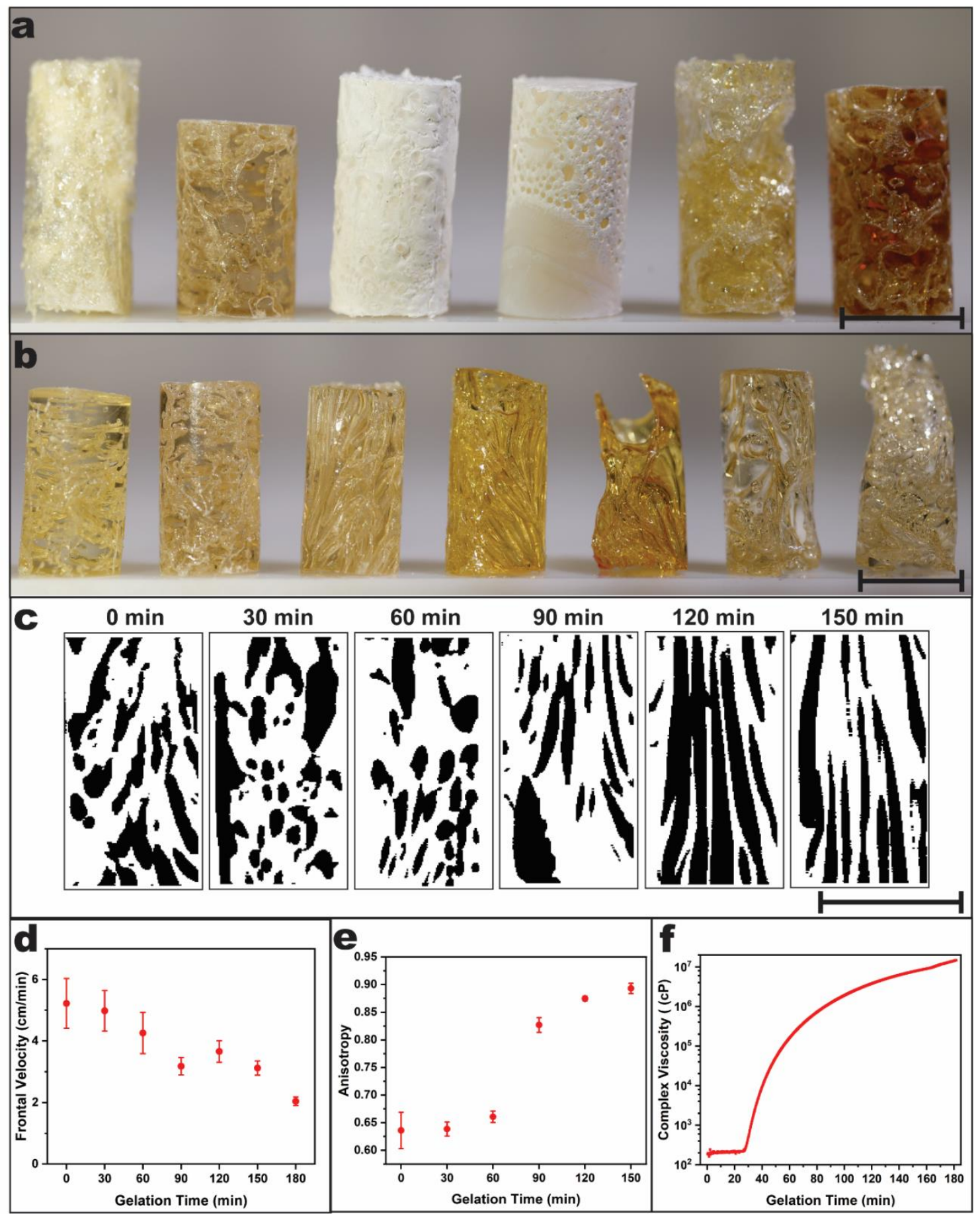

Figure 1. a. DCPD foams using different blowing agents at $20 \mathrm{wt} \%$, from left to right: $n$-pentane, cyclohexane, isopropanol, n-butanol, dichloromethane, and chloroform. b. DCPD foams using different mass fraction of cyclohexane, from left to right: $10 \mathrm{wt} \%, 20 \mathrm{wt} \%, 30 \mathrm{wt} \%, 40 \mathrm{wt} \%, 50 \mathrm{wt} \%, 60 \mathrm{wt} \%$, and 70 wt\%. For a, b; [GC2]: $0.60 \mathrm{mM}$, [TBP]: $0.38 \mathrm{mM}$, [PCH]: 5.1 vol\% c. Cross section of DCPD/cyclohexane $(80 / 20)$ foams from X-ray microtomography as a function of gelation time, where the voids are depicted in black. $\mathbf{d}$. Frontal velocity as a function of gelation time of a DCPD/cyclohexane (80/20) resin. e. Anisotropy as a function of gelation time of a DCPD/cyclohexane (80/20) resin. $\mathbf{f}$. Viscosity change as a function of time for a DCPD/cyclohexane (80/20) resin. For c, d, e, f; [GC2]: $0.30 \mathrm{mM}$, [TBP]: $0.30 \mathrm{mM},[\mathrm{PCH}]: 2.5$ vol\%. Scale bars $10 \mathrm{~mm}$. 


\section{Full Factorial Design Study}

We employed a full factorial design to study the influence of blowing agent, concentration, and gelation time on the volume fraction of the solid phase and the anisotropy of the cellular structure. The full factorial design led to 100 total combinations of conditions, which we used to prepare the foams via FROMP. Afterwards, we imaged each sample using X-ray microcomputed tomography, followed by image reconstruction, processing, and calculation of volume fraction and anisotropy. ${ }^{[24]}$ Then, we used compression testing to investigate the compression modulus of the samples. Finally, the statistical analysis was done using blowing agent, concentration, and gelation time as input, and volume fraction anisotropy, and compression modulus as outputs. From the variable combination analysis, we observed significant correlations between the blowing agent concentration and the cellular volume fraction, between the gelation time and the anisotropy, and between the compression modulus and blowing agent boiling point and concentration (Table S1).

Further analysis of the correlation plots showed that volume fraction of the solid phase and compression modulus increased with the blowing agent's boiling point, presumably caused by the higher temperature required to achieve a high vapor pressure from the blowing agent. Also, we observed that solid volume fraction and compression modulus decreased by increasing blowing agent concentration due to the lower amount of monomer used in the polymerization. Anisotropy increased with longer gelation times, supporting our previous observation of the correlation between foams cellular structure 
and resin viscosity (Figure S10). Relation analysis of the output parameters shows no significant correlation between anisotropy with compression modulus or volume fraction. Moreover, compression modulus increases as volume fraction increases (Figure S11). We constructed a heat plot between compression modulus, anisotropy, and volume fraction of the foams to aid in the design materials with specific characteristics, e.g., a low modulus foam with high porosity and high alignment is obtained using $20 \mathrm{wt} \%$ of $n$ pentane initiated at 90 min (Figure 2a and SI interactive plot). We observed regions of high and low modulus, anisotropy, and volume fraction, demonstrating the selected input parameters produce cellular solids with a wide range of properties.

Subsequently, to investigate if different resin formulations produce similar foams, we applied a hierarchical cluster analysis to the output variables, using an algorithm that grouped samples with similar mechanical properties and structural characteristics. We identified ten clusters and obtained the average of the variables for each cluster (Figure 2b). Within each cluster, we found that materials having similar output variables resulted from formulations with different combinations of input variables (blowing agents, concentrations and gelation times). For instance, all the formulations in the first cluster produced foams with low alignment of the cellular structure, medium porosities, and low compression modulus; these foams were derived from formulations differing in their input variables. Finally, four samples were significantly different enough that they did not cluster until the normalized distance between neighbors reached 0.4 , because their characteristics were at the edges of the full factorial design: high anisotropy, high volume fraction, and high compression modulus (cluster 5 and 6) or low anisotropy, low volume 
fraction, and low compression modulus (cluster 9 and 10). The statistical and hierarchical clustering analyses showed transitions from isotropic to anisotropic cellular structures, low to high porosities, and hard to soft foams. Blowing agent and concentration have a significant effect on the foam porosity and compression modulus while the resin viscosity, expressed as gelation time, had a significant effect on the cellular alignment.

We replicated three aleatory formulations, two porous and one non-porous, of the factorial design to demonstrate the reproducibility of the method. The anisotropy and volume fraction variability for the two porous formulations was between $1.6 \%$ and $2.8 \%$, respectively, which indicates that the formulations give reproducible structures (Figure S12).

a

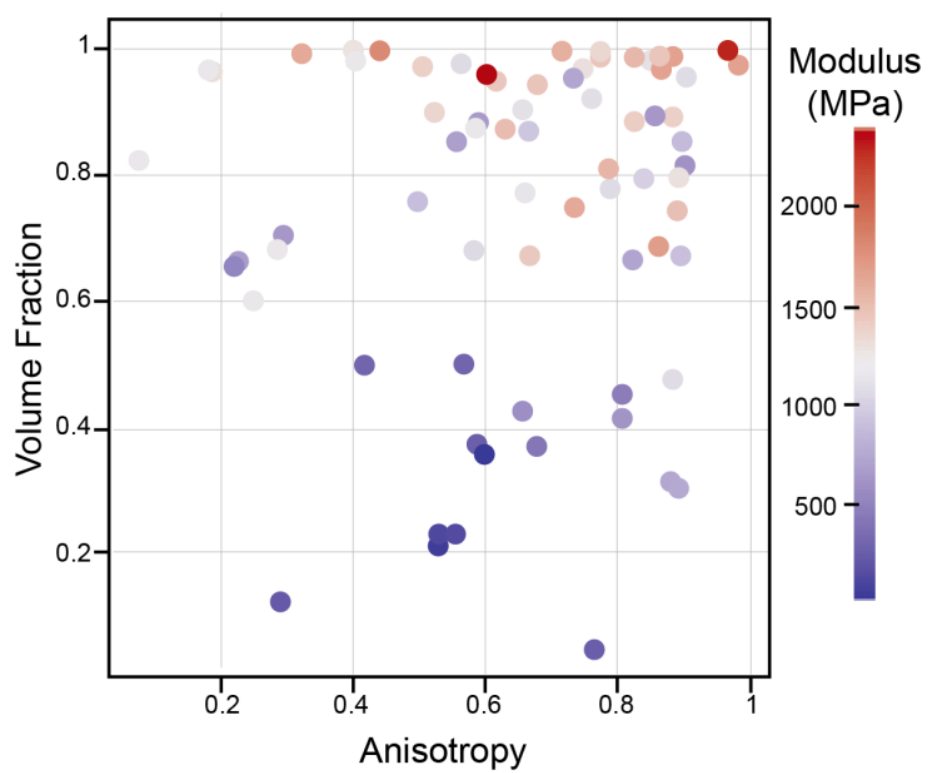

b

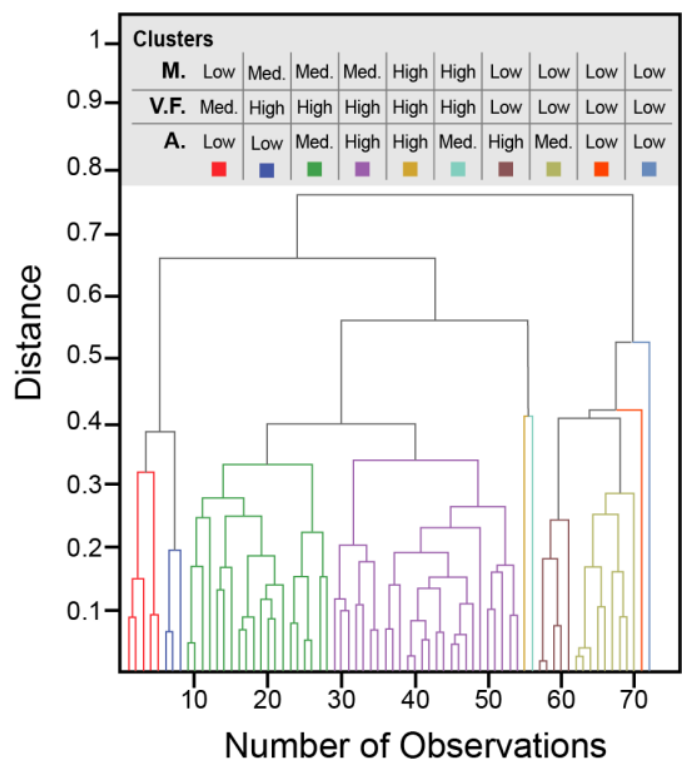

Figure 2. a. Cellular structure anisotropy, solid volume fraction, and foam compression modulus heat plot. b. Dendrogram obtained from hierarchical cluster analysis of anisotropy, volume fraction, and compression modulus. Cluster method: group average, distance type: Euclidean, variables: normalized to $(0,1)$. Clusters variables; A: Anisotropy, VF: Volume Fraction, CM: Compression Modulus. Figure 2a interactive plot. 
Infiltration of liquid revealed the connectivity and alignment in a foam with high anisotropy. We used the $10 \mathrm{wt} \% \mathrm{n}$-pentane initiated at $60 \mathrm{~min}$ formulation which has an anisotropy value of 0.89 . To test the foams' ability to transport liquids, we added $0.2 \mathrm{~mL}$ of silicon oil tainted with red colorant on top of the monolith and allowed it to infuse through the structure. We observed a rapid absorption of the oil in the foam followed by a migration through the structure, which is due to the high anisotropy and connectivity of the compartments in the foam (Figure $3 a$ and $3 b$ ). Furthermore, a solid with isolated pores in a radially aligned cellular structure was prepared using the same formulation but triggering the reaction in the center, rather than at the bottom. Hence, our methodology allows the fabrication of foams with diverse topologies, cellular orientations and shapes (Figure 3c).

Infiltration experiments demonstrate a non-deterministic but robust method to fabricate structural foams capable of transporting liquids, which will open the way for using directional foams in transport-dependent applications. We also demonstrated the radial distribution of cellular structures, which lays the foundation for an alternative construction of vascular systems. Previous work in our laboratory has demonstrated a deterministic FP approach to fabricate vascularized structures i.e., using sacrificial fibers that depolymerize from the heat of the frontal reaction. ${ }^{[25]}$ For some applications, creating anisotropic cellular solids by methods described herein may simplify materials processing without limiting performance attributes. 


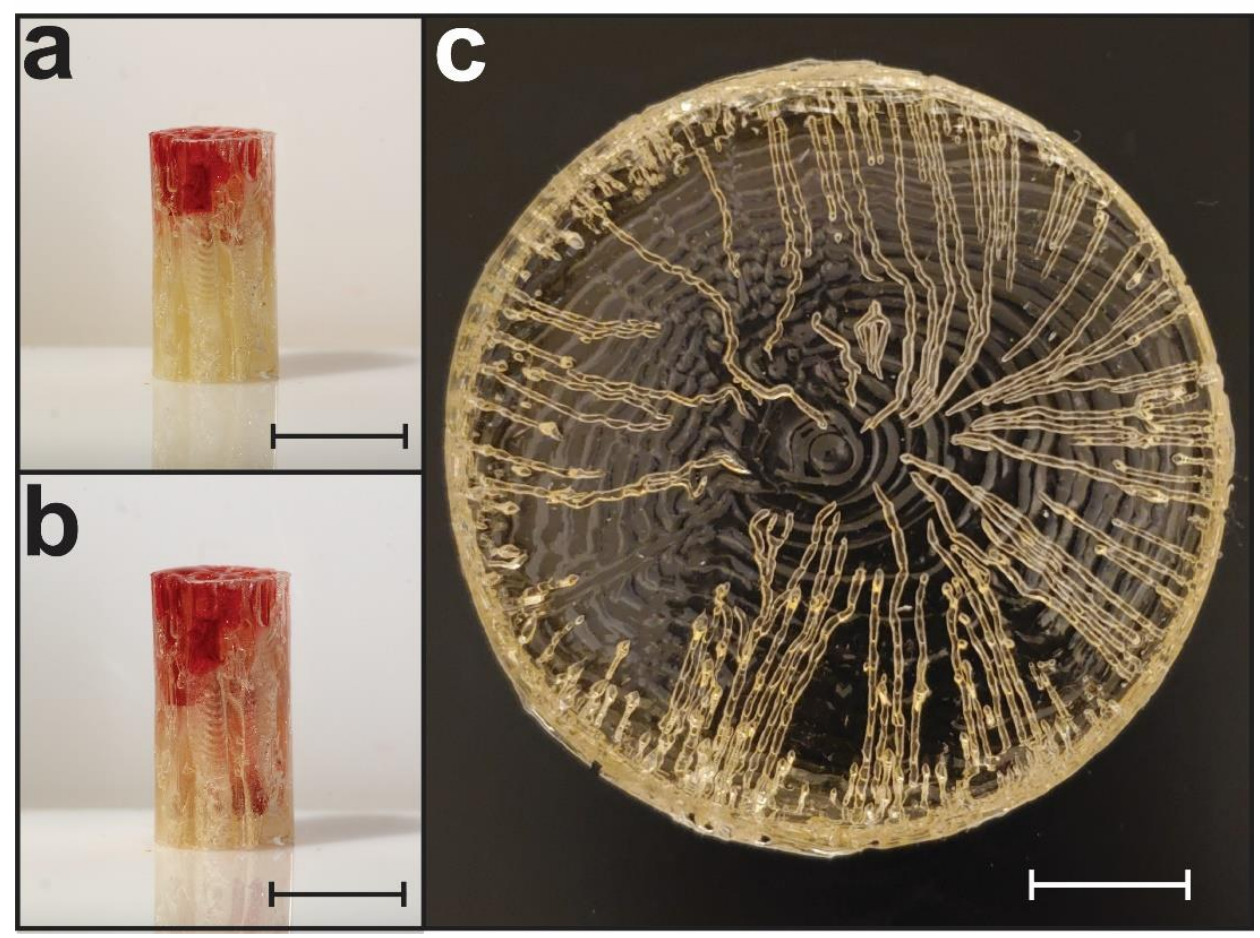

Figure 3. Infiltration of dye-colored silicon oil into a DCPD foam at $\mathbf{a} . t=0 \mathrm{~s}, \mathbf{b} . t=10 \mathrm{~s}, \mathbf{c}$. DCPD foam obtained in a petri dish; reaction triggered in the center of the resin. [DCPD]: $90 \mathrm{wt} \%$, [n-pentane]: $10 \mathrm{wt} \%$, Gelation time: $60 \mathrm{~min}$, [GC2]: $0.30 \mathrm{mM}$, [TBP]: $0.30 \mathrm{mM}$, [PCH]: 2.5 vol\%. Scale bars $10 \mathrm{~mm}$.

\section{Conclusions}

We used a fast, efficient, and low-cost FP method to introduce desirable morphological features in poly-DCPD structural foams. The formation of anisotropic structure was hypothesized based on physicochemical principles of the frontal process. Our initial rationale considered the thermomechanical gradient fields of temperature and viscosity at the frontal boundary with void formation fed by the thermally driven vaporization of a physical blowing agent. However, we discovered that the rheology of the gel-forming resin was an important attribute in realizing anisotropic structure. Through extensive experimentation, we demonstrated that the foam morphology is easily tuned by changing the blowing agent, concentration, and gelation time. Specifically, the alignment of the foam cellular structure was dependent on the viscosity of the resin. We used a full factorial 
design to establish relations between blowing agents, concentrations, and gelation times with the volume fractions and anisotropies of the foams. Finally, we showed that radially aligned foams can transport liquids over large distances in controlled directions through the polymeric solids. The method opens opportunities for the non-deterministic fabrication of novel porous structural foams for specific applications. We see this foaming method as expanding the toolbox of the materials chemist using the physicochemical principles to achieve form and function in a single step.

\section{References}

[1] N. Mills, Polymer Foams Handbook, Elsevier Ltd, 2007.

[2] T. Weigel, G. Schinkel, A. Lendlein, Expert Rev. Med. Devices 2006, 3, 835.

[3] E. Aram, S. Mehdipour-Ataei, Int. J. Polym. Mater. Polym. Biomater. 2016, 65, 358.

[4] N. A. Campbell, J. B. Reece, Biology, Benjamin Cummings, San Francisco, CA, USA, 2002.

[5] K. Gładyszewski, K. Groß, A. Bieberle, M. Schubert, M. Hild, A. Górak, M. Skiborowski, Chem. Eng. Sci. 2021, 230, 116176.

[6] Q. Gong, J. Wu, X. Gong, Y. Fan, H. Xia, RSC Adv. 2013, 3, 3241.

[7] S. Deville, Scr. Mater. 2018, 147, 119.

[8] Q. Chen, P.-F. Cao, R. C. Advincula, Adv. Funct. Mater. 2018, 28, 1800631.

[9] S. Wang, G. Wang, X. Zhang, Y. Tang, J. Wu, X. Xiang, X. Zu, Q. Yu, Carbon N. Y. 2017, 120, 103.

[10] J. Yang, W. Yang, W. Chen, X. Tao, Prog. Polym. Sci. 2020, 109, 101289.

[11] C. D. Christiansen, K. K. Nielsen, R. Bjørk, Rev. Sci. Instrum. 2020, 91, 033904.

[12] J. A. Pojman, Q. Tran-Cong-Miyata, Nonlinear Dynamics with Polymers, WileyVCH Verlag GmbH \& Co. KGaA, Weinheim, Germany, 2010.

[13] J. D. Mota-Morales, M. C. Gutiérrez, M. L. Ferrer, R. Jiménez, P. Santiago, I. C. Sanchez, M. Terrones, F. Del Monte, G. Luna-Bárcenas, J. Mater. Chem. A 2013, 1, 3970.

[14] K. Bansal, J. A. Pojman, D. Webster, M. Quadir, ACS Macro Lett. 2020, 14, 169.

[15] G. D. Lu, Q. Z. Yan, C. C. Ge, Polym. Int. 2007, 56, 1016. 
[16] Q. Z. Yan, G. D. Lu, W. F. Zhang, X. H. Ma, C. C. Ge, Adv. Funct. Mater. 2007, 17, 3355.

[17] M. Howe-Grant, Ed. , Encyclopedia of Chemical Technology, Wiley-Interscience, New York, 1996.

[18] A. Mariani, S. Fiori, Y. Chekanov, J. A. Pojman, Macromolecules 2001, 34, 6539.

[19] I. D. Robertson, L. M. Dean, G. E. Rudebusch, N. R. Sottos, S. R. White, J. S. Moore, ACS Macro Lett. 2017, 6, 609.

[20] I. D. Robertson, M. Yourdkhani, P. J. Centellas, J. E. Aw, D. G. Ivanoff, E. Goli, E. M. Lloyd, L. M. Dean, N. R. Sottos, P. H. Geubelle, J. S. Moore, S. R. White, Nature 2018, 557, 223.

[21] S. Kovačič, C. Slugovc, Mater. Chem. Front. 2020, 4, 2235.

[22] B. A. Suslick, K. J. Stawiasz, J. E. Paul, N. R. Sottos, J. S. Moore, Macromolecules 2021, 54, 5117.

[23] C. T. Rueden, J. Schindelin, M. C. Hiner, B. E. DeZonia, A. E. Walter, E. T. Arena, K. W. Eliceiri, BMC Bioinformatics 2017, 18, 529.

[24] M. Doube, M. M. Klosowski, I. Arganda-Carreras, F. P. Cordelières, R. P. Dougherty, J. S. Jackson, B. Schmid, J. R. Hutchinson, S. J. Shefelbine, Bone 2010, 47, 1076.

[25] M. Garg, J. E. Aw, X. Zhang, P. J. Centellas, L. M. Dean, E. M. Lloyd, I. D. Robertson, Y. Liu, M. Yourdkhani, J. S. Moore, P. H. Geubelle, N. R. Sottos, Nat. Commun. 2021, 12, 1.

\section{Acknowledgments}

This work was supported by Air Force Office of Scientific Research under award number FA9550-20-1-0194 and the National Science Foundation under award number NSF CMMI 19-33932. This work was conducted in part at the Microscopy Suite of the Beckman Institute for Advanced Science and Technology at the University of Illinois at UrbanaChampaign (UIUC-BI-ITG). The authors acknowledge the Beckman Institute at the University of Illinois, Urbana-Champaign, as well as Justine Paul, Polette Centellas and Nil Parikh for assistance with the foam's characterization. Katherine Stawiasz, Yunyan Sun, Benjamin Suslick, William Neary, and Julian Cooper are thanked for insightful discussions. 
